\title{
Minimal number of periodic points of smooth boundary-preserving self-maps of simply-connected manifolds
}

\author{
Grzegorz Graff $^{1}$ (D) · Jerzy Jezierski
}

Received: 3 May 2016 / Accepted: 4 October 2016 / Published online: 15 October 2016

(C) The Author(s) 2016. This article is published with open access at Springerlink.com

\begin{abstract}
Let $M$ be a smooth compact and simply-connected manifold with simplyconnected boundary $\partial M, r$ be a fixed odd natural number. We consider $f$, a $C^{1}$ self-map of $M$, preserving $\partial M$. Under the assumption that the dimension of $M$ is at least 4 , we define an invariant $D_{r}(f ; M, \partial M)$ that is equal to the minimal number of $r$-periodic points for all maps preserving $\partial M$ and $C^{1}$-homotopic to $f$. As an application, we give necessary and sufficient conditions for a reduction of a set of $r$-periodic points to one point in the $C^{1}$-homotopy class.
\end{abstract}

Keywords Periodic points · Boundary-preserving maps $\cdot$ Nielsen number $\cdot$ Fixed point index $\cdot$ Smooth maps

Mathematics Subject Classification (2000) Primary 37C25 · 55M20 · Secondary 37C05

\section{Introduction}

Finding minimal number of $r$-periodic points in the homotopy class (for a fixed $r$ ) is an important challenge in modern homotopy periodic point theory, with an increasing number of valuable results obtained in the last decade in many particular cases [1-7].

On the other hand, the smooth case in which smooth homotopies are considered, turned out to be essentially different from the continuous one (cf. [8]). First authors who observed a difference between smooth and continuous category in Nielsen theory were Brown, Greene and Schirmer for $r=1$ i.e. for fixed points and in the relative case [9] (see also [10-13]

Grzegorz Graff

graff@mif.pg.gda.pl

Jerzy Jezierski

jezierski@acn.waw.pl

1 Faculty of Applied Physics and Mathematics, Gdansk University of Technology,

Narutowicza 11/12, 80-233 Gdańsk, Poland

2 Institute of Applications of Mathematics, Warsaw University of Life Sciences (SGGW),

Nowoursynowska 159, 00-757 Warsaw, Poland 
for related results). These authors considered a smooth manifold $M$ with boundary $\partial M$ and $\phi$, a smooth self-map of $\partial M$. They asked a question whether it is possible to extend $\phi$ from $\partial M$ to $M$ without introducing any more fixed points. It turned out that the answer depends whether one considers smooth or continuous extensions: there are smooth maps $\phi$ which admit continuous extensions to $M$ with no fixed points on $M \backslash \partial M$, but such that every smooth extension has a fixed point in $M \backslash \partial M$. This rather unexpected result showed that there is a differences between smooth and continuous case in fixed point theory, even if there was no such difference in classical Nielsen theory i.e. for the non-relative case (cf. [14]).

In this paper we raise more general question related to minimization the number of periodic points instead of the number of fixed points in the smooth category. We consider maps of pairs $f:(M, \partial M) \rightarrow(M, \partial M)$ and ask for minimal number of periodic points in the smooth (i.e. $C^{1}$ ) homotopy class (preserving the boundary) of $f$. Let us point out that we consider all $C^{1}$ maps, generalizing the approach presented in [9] were the class of transversal maps was examined. On the other hand, we confine ourselves to the case in which both $M$ and $\partial M$ are simply-connected. The reason for that is the following: there are two obstacles to minimize the number of periodic points for $C^{1}$ maps. One of them comes from the Reidemeister relations and the second results from the restrictions on the sequence of local indices of iterations of a $C^{1}$ map. It would be difficult (also from the computational point of view) to follow the restrictions that come from the both conditions simultaneously, and thus we analyze the situation in which the fundamental group is trivial, so the Reidemeister relations disappear. Then the only obstacle that we have to control is related to the forms of local indices of iterations and we can apply the topological methods developed in [15] that were used to minimize the number of periodic points for $C^{1}$ self-maps of simply-connected manifold without boundary. As these technics work only in dimension at least 3 we have to assume that $\operatorname{dim} \partial M \geq 3$. We consider only odd $r$ : the case of even $r$ is conceptually analogous, but the forms of indices of iterations are much more complicated and thus analyzing the even case would overshadowed our main idea.

The paper is organized in the following way. In Sect. 2 we define the invariant $D_{r}(f ; M, \partial M)$ and prove that it is equal to the minimal number of periodic points in the $C^{1}$-homotopy class of $f$ (for homotopies preserving the boundary).

In Sect. 3 we consider $(f, \bar{f}):(M, \partial M) \rightarrow(M, \partial M), x_{0} \in \partial M$ a fixed point of $f$ and find all possible forms of pairs of sequences $\left(\operatorname{ind}\left(f^{n}, x_{0}\right) \text {, ind }\left(\bar{f}, x_{0}\right)\right)_{n}$ (Theorem 3.15), which is necessary for effective computation of $D_{r}(f ; M, \partial M)$. In the last section we show our invariant in action, finding necessary and sufficient conditions for a reduction of a set of $r$-periodic points to one point and illustrating this case in the example of self-maps of a 6-dimensional closed ball.

\section{Definition of the invariant $D_{r}(f ; M, \partial M)$}

Let $r$ be a fixed natural number, $M$ be a smooth compact and simply-connected manifold with $\operatorname{dim} M \geq 4$, such that its boundary $\partial M$ is also simply-connected. We consider a $C^{1}$ map $f: M \rightarrow M$, preserving boundary: $f(\partial M) \subset \partial M$. Thus, we may consider maps of a pair $(M, \partial M),(f, \bar{f}):(M, \partial M) \rightarrow(M, \partial M)$.

Let us emphasize that the pair $(f, \bar{f})$ is determined by $f$, so we will sometimes overuse the notation identifying $f$ and $(f, \bar{f})$. 
In this section we define an invariant $D_{r}(f ; M, \partial M)$ of a pair and we will show that

$$
D_{r}(f ; M, \partial M)=\min _{(g, \bar{g}) \sim(f, \bar{f})} \# \operatorname{Fix}\left(g^{r}\right),
$$

where $\stackrel{s}{\sim}$ means that $(g, \bar{g})$ is $C^{1}$-homotopic to $(f, \bar{f})$ as a map of a pair $(M, \partial M)$.

Remark 2.1 We will assume for the rest of the paper that $\operatorname{Fix}\left(f^{r}\right)$ is a finite set, because we could always approximate our map by a map with a finite set of $r$-periodic points (cf. [16]).

Remark 2.2 The minimum given in (2.1) does not change if we consider only such pairs $(g, \bar{g})$ that the only $r$-periodic points of $g$ (and so $\bar{g}$ ) are fixed points (which results from the similar considerations as those given in Sect. 4 in [17], see also Lemma 4.8 in [15]). This statement is true for each $r$ in dimension at least 4 and for odd $r$ also in dimension 3. In other words, we could seek for the minimum over the maps having only fixed points up to the $r$ th iteration (but we have to assume that $r$ is odd and $\operatorname{dim} \partial M \geq 3$ ).

Definition 2.3 A sequence of integers $\{c(n)\}_{n=1}^{\infty}$ is called a $D D^{m}(1)$ sequence if there are: a $C^{1}$ map $\psi: U \rightarrow \mathbb{R}^{m}$, where $U \subset \mathbb{R}^{m}$ is open; and $p$, an isolated fixed point of $\psi$, such that $c(n)=\operatorname{ind}\left(\psi^{n}, p\right)$. A finite sequence $\{c(n)\}_{n \mid r}$ will be called a $D D^{m}(1 \mid r)$ sequence if this equality holds for all $n$ with $n \mid r$, where $r$ is fixed.

We introduce the following notations:

$$
\begin{aligned}
& \mathbb{R}_{+}^{m+1}=\left\{(x, t) \in \mathbb{R}^{m} \times \mathbb{R}: t \geq 0\right\}, \\
& \mathbb{R}_{-}^{m+1}=\left\{(x, t) \in \mathbb{R}^{m} \times \mathbb{R}: t \leq 0\right\}, \\
& \mathbb{R}_{0}^{m+1}=\left\{(x, t) \in \mathbb{R}^{m} \times \mathbb{R}: t=0\right\} .
\end{aligned}
$$

We will consider $C^{1}$ maps of a pair

$$
(f, \bar{f}):\left(\mathbb{R}_{+}^{m+1}, \mathbb{R}_{0}^{m+1}\right) \rightarrow\left(\mathbb{R}_{+}^{m+1}, \mathbb{R}_{0}^{m+1}\right),
$$

by a $C^{1}$ map we understand a map which has continuous partial derivatives of degree 1 , where for a point $x_{0} \in \mathbb{R}_{0}^{m+1}$ we consider right derivatives $\frac{\partial_{+} f_{i}\left(x_{0}\right)}{\partial t}, i=1, \ldots, m+1$.

Definition 2.4 A pair of sequences of integers $\left(\{c(n)\}_{n=1}^{\infty},\{\bar{c}(n)\}_{n=1}^{\infty}\right)$ is called a $\partial D D^{m}(1)$ pair of sequences if there is an isolated fixed point $p \in \mathbb{R}_{0}^{m+1}$, its open neighborhood $U_{+}^{m+1} \subset \mathbb{R}_{+}^{m+1}$, and a pair of $C^{1} \operatorname{maps}(\phi, \bar{\phi}):\left(U_{+}^{m+1}, U_{0}^{m+1}\right) \rightarrow\left(\mathbb{R}_{+}^{m+1}, \mathbb{R}_{0}^{m+1}\right)$ $\left(U_{0}^{m+1}=U_{+}^{m+1} \cap \mathbb{R}_{0}^{m+1}\right)$ such that $(c(n), \bar{c}(n))=\left(\operatorname{ind}\left(\phi^{n}, p\right)\right.$, ind $\left.\left(\bar{\phi}^{n}, p\right)\right)$. A pair of finite sequences $\left(\{c(n)\}_{n \mid r},\{\bar{c}(n)\}_{n \mid r}\right)$ will be called a $\partial D D^{m}(1 \mid r)$ pair of sequences if this equality holds for all $n$ with $n \mid r$, where $r$ is fixed.

\subsection{Construction of the invariant}

Let $(f, \bar{f}):(M, \partial M) \rightarrow(M, \partial M)$, we consider maps $(g, \bar{g})$ of the pair $(M, \partial M) C^{1}$ homotopic to $(f, \bar{f})$ and (by Remarks 2.1 and 2.2) such that

$$
\operatorname{Fix}\left(g^{r}\right)=\left\{x_{1}, \ldots, x_{\alpha}, y_{1}, \ldots, y_{\beta}\right\}
$$

is finite and consists only of fixed points of $g$, where $x_{j} \in M \backslash \partial M, y_{i} \in \partial M$. 
The above yields the following decomposition of the sequences of Lefschetz numbers of iterations of $f$ and $\bar{f}$ :

$$
L\left(\bar{f}^{n}\right)=L\left(\bar{g}^{n}\right)=\sum_{i=1}^{\beta} \bar{c}_{i}(n),
$$

where $\bar{c}_{i}(n)=\operatorname{ind}\left(\bar{g}^{n}, y_{i}\right)$.

$$
L\left(f^{n}\right)=L\left(g^{n}\right)=\sum_{j=1}^{\alpha} d_{j}(n)+\sum_{i=1}^{\beta} c_{i}(n),
$$

where $d_{j}(n)=\operatorname{ind}\left(g^{n}, x_{j}\right)$ and $c_{i}(n)=\operatorname{ind}\left(g^{n}, y_{i}\right)$.

Definition 2.5 Let $r$ be fixed and $L\left(f^{n}\right)=\sum_{j=1}^{\alpha} d_{j}(n)+\sum_{i=1}^{\beta} c_{i}(n)$ and $L\left(\bar{f}^{n}\right)=$ $\sum_{i=1}^{\beta} \bar{c}_{i}(n)$, for $n \mid r$, where each $d_{j}$ is a $D D^{m+1}(1 \mid r)$ sequence and each $\left(c_{i}, \bar{c}_{i}\right)$ is a $\partial D D^{m}(1 \mid r)$ pair of sequences. Each such decomposition of Lefschetz numbers determines a sum $\alpha+\beta$. We define the number $D_{r}(f ; M, \partial M)$ as the smallest $\alpha+\beta$ which can be obtained in this way.

\subsection{Main theorem}

In this section we will prove one of the main results of the paper: Theorem 2.10. First, we will introduce two procedures, which make it possible to create and remove fixed or periodic points in a homotopy class.

The Creating Procedure enables one to create an additional fixed point in the $C^{1}$-homotopy class of $f$, by a use of a homotopy $f_{t}$ which is constant near periodic points of $f$ (up to the given period $r$ ) and such that $f_{1}^{r}$ near the new fixed point is given by any prescribed formula.

Theorem 2.6 (Smooth Creating Procedure, compare [8] Theorem 4.5) Let $\operatorname{dim} M \geq 3$, given a fixed $r \in \mathbb{N}$ and a $C^{1}$ map $f: M \rightarrow M$, such that $\operatorname{Fix}\left(f^{r}\right)$ is finite and let point $x_{0} \notin \operatorname{Fix}\left(f^{r}\right)$. Then there is a homotopy $\left\{f_{t}\right\}_{0 \leq t \leq 1}$ satisfying:

(1) $f_{0}=f$.

(2) $\left\{f_{t}\right\}$ is constant in a neighborhood of $\operatorname{Fix}\left(f^{r}\right)$.

(3) $f_{1}\left(x_{0}\right)=x_{0}$ and $\left\{x_{0}\right\}$ is isolated in Fix $\left(f_{1}^{r}\right)$.

(4) $f_{1}$ realizes given $D D^{m}(1 \mid r)$ sequence $\left\{c_{n}\right\}_{n \mid r}$ on $x_{0}$, i.e. $f_{1}$ could be any $C^{1}$ map in a neighborhood of $x_{0}$ such that $c_{n}=\operatorname{ind}\left(f_{1}^{n}, x_{0}\right)$ for $n \mid r$.

Due to the next lemma it is possible to cancel, in the homotopy class, invariant subsets of periodic points which have indices of iterations equal to zero.

Lemma 2.7 (Cancelling Lemma, [18] Lemma 5.2) Let $f$ be a continuous self-map of $M$. Suppose that $S \subset \operatorname{Fix}\left(f^{r}\right)$ satisfies:

(1) $S$ is finite and $f$-invariant i.e. $f(S)=S$.

(2) $\operatorname{Fix}\left(f^{r}\right) \backslash S$ is compact.

(3) $\operatorname{ind}\left(f^{n}, \operatorname{Fix}\left(f^{n}\right) \backslash S\right)=0$ for all $n \mid r$.

Then there is a homotopy $f_{t}$, starting from $f_{0}=f$, constant in a neighborhood of $S$ and such that $\operatorname{Fix}\left(f_{1}^{r}\right)=S$.

The next lemma enables us to construct homotopies preserving the boundary, which we will use in the proof of Theorem 2.10. 
Lemma 2.8 Let $B_{0} \subset \mathbb{R}_{0}^{m+1}$ be an open ball centered at 0 . Let $f, g: \operatorname{cl}\left(B_{0}\right) \times[0, \varepsilon] \rightarrow$ $\mathbb{R}_{+}^{m+1}$ be continuous maps preserving $\mathbb{R}_{0}^{m+1}$ (i.e. $f(x, 0)=\left(x^{\prime}, t^{\prime}\right)$ implies $t^{\prime}=0$ and $g\left(x^{\prime}, 0\right)=\left(x^{\prime}, t^{\prime}\right)$ implies $\left.t^{\prime}=0\right)$. We fix a number $\varepsilon>0$. Then there are: a homotopy $H: \operatorname{cl}\left(B_{0}\right) \times[0, \varepsilon] \times[0,1]$, an open set $V_{0} \subset B_{0}$ containing 0 and a number $\varepsilon^{\prime}>0$ satisfying:

(1) $H(x, t, 0)=f(x, t)$ for $x \in \operatorname{cl}\left(B_{0}\right) \times[0, \varepsilon]$,

(2) $H(x, t, 1)=g(x, t)$ for $x \in \operatorname{cl}\left(V_{0}\right) \times\left[0, \varepsilon^{\prime}\right]$,

(3) $H(x, t, \tau)=f(x, t)$ for all $\tau \in[0,1]$ and $x \in \partial\left(B_{0} \times[0, \varepsilon]\right)$,

(4) $H_{\tau}$ preserves $\mathbb{R}_{0}^{m+1}$ for each $\tau$.

Proof We take as $V_{0}$ any open set satisfying $0 \in V_{0} \subset \operatorname{cl}\left(V_{0}\right) \subset B_{0}$ and as $\varepsilon^{\prime}$ any number $0<\varepsilon^{\prime}<\varepsilon$. For two disjoint closed subsets in $\mathbb{R}_{+}^{m+1}: \operatorname{cl}\left(V_{0}\right) \times\left[0, \varepsilon^{\prime}\right]$ and $\partial\left(B_{0} \times[0, \varepsilon]\right)$ we take a smooth Urysohn function $\eta: \operatorname{cl}\left(B_{0}\right) \times[0, \varepsilon] \rightarrow[0,1](\eta(x, t)=1$ for $(x, t) \in$ $c l\left(V_{0}\right) \times\left[0, \varepsilon^{\prime}\right], \eta(x, t)=0$ for $\left.(x, t) \in \partial\left(B_{0} \times[0, \varepsilon]\right)\right)$. Finally, we define

$$
H(x, t, \tau)=(1-\tau \eta(x, t)) \cdot f(x, t)+\tau \eta(x, t) \cdot g(x, t) .
$$

Corollary 2.9 Let $(f, \bar{f}):(M, \partial M) \rightarrow(M, \partial M)$ satisfy $f\left(x_{0}\right)=x_{0} \in \partial M$ and let $g$ be a map defined in a neighborhood of $x_{0}$ in $M$. Then for any neighborhood $U \subset M$ of $x_{0}$ there is a homotopy $H: M \times[0,1] \rightarrow M$ with the support in $U$, preserving $\partial M$ and such that $H(x, 0)=f(x)$ for $x \in M, H(x, 1)=g(x)$ in some neighborhood of $x_{0} \in M$. Notice that if $f, g$ and $\eta$ are $C^{1}$ maps then so is $H(\cdot, 1)$.

Theorem 2.10 Let $M$ be a smooth compact and simply-connected manifold of dimension $m \geq 4$ with simply-connected boundary $\partial M$ and $r \in \mathbb{N}$ a fixed odd number. Let $(f, \bar{f})$ : $(M, \partial M) \rightarrow(M, \partial M)$ be a $C^{1}$ map of a pair. Then,

$$
D_{r}(f ; M, \partial M)=\min _{(g, \bar{g}) \sim(f, \bar{f})} \# \operatorname{Fix}\left(g^{r}\right) .
$$

Proof First of all, let us notice that the minimum is greater or equal to $D_{r}(f ; M, \partial M)$ by the equalities (2.3) and (2.4).

To show the inverse inequality we decompose

$$
\begin{aligned}
& L\left(\bar{f}^{n}\right)=\sum_{i=1}^{\beta} \bar{c}_{i}(n), \\
& L\left(f^{n}\right)=\sum_{j=1}^{\alpha} d_{j}(n)+\sum_{i=1}^{\beta} c_{i}(n),
\end{aligned}
$$

where $n \mid r$, each $\left(c_{i}, \bar{c}_{i}\right)$ is a $\partial D D^{m}(1 \mid r)$ pair of sequences and each $d_{j}$ is a $D D^{m+1}(1 \mid r)$ sequence. We will prove that for any sum of $(\alpha+\beta)$ sequences given in (2.5) and (2.6) we are able to find a $C^{1}$ map $f_{1} C^{1}$-homotopic to $f$, by a homotopy preserving the boundary, with \#Fix $\left(f_{1}^{r}\right)=\alpha+\beta$.

The proof will be done in three steps: first we minimize the number of $r$-periodic points in the boundary $\partial M$, next we realize given indices near the boundary fixed points in the whole $M$ and in the last step we minimize the number of $r$-periodic points in $\operatorname{Int} M$ by a homotopy which is constant near $\partial M$.

Consider $(f, \bar{f}):(M, \partial M) \rightarrow(M, \partial M)$. Abusing the notation we will use below the same letters for the homotopic maps obtained after consecutive applications of the Creating and the Cancelling Procedures. 
Step 1. Minimization of the number of $r$-periodic points in the boundary $\partial M$.

We deform $f$ to get $f(x, t)=(\bar{f}(x), t)$ in a collar $\partial M \times[0,1)$. Then we choose points $y_{1}, \ldots, y_{\beta} \in \partial M$ and their ball neighborhoods $B_{i}$ satisfying $c l B_{i} \cap c l B_{j}=\emptyset$ for $i \neq j$.

Now, we consider maps $\bar{g}_{i}$ such that $\left(g_{i}, \bar{g}_{i}\right):\left(B_{i} \times[0, \varepsilon), B_{i} \times\{0\}\right) \rightarrow(M, \partial M)$ is a $C^{1}$ map realizing ind $\left(\bar{g}_{i}^{n}, y_{i}\right)=\bar{c}_{i}(n)$, ind $\left(g_{i}^{n}, y_{i}\right)=c_{i}(n)$ for $n \mid r, i=1, \ldots, \beta$.

Applying the Creating Procedure to $\bar{f}: \partial M \rightarrow \partial M$ we may deform $\bar{f}$ to get $\bar{f}(x)=\bar{g}_{i}(x)$ for $x \in B_{i}$. We may replace each $B_{i}$ by a smaller ball neighborhood of $y_{i}$ so that $y_{i}$ is a unique $r$-periodic point of $\bar{f}$ in $B_{i}$. Then, for $n \mid r$

$$
\begin{aligned}
L\left(\bar{f}^{n}\right) & =\operatorname{ind}\left(\bar{f}^{n},\left\{y_{1}, \ldots, y_{\beta}\right\}\right)+\operatorname{ind}\left(\bar{f}^{n}, \partial M \backslash\left\{y_{1}, \ldots, y_{\beta}\right\}\right) \\
& =\sum_{i=1}^{\beta} \operatorname{ind}\left(\bar{f}^{n}, y_{i}\right)+\operatorname{ind}\left(\bar{f}^{n}, \partial M \backslash\left\{y_{1}, \ldots, y_{\beta}\right\}\right) \\
& =\sum_{i=1}^{\beta} \bar{c}_{i}(n)+\operatorname{ind}\left(\bar{f}^{n}, \partial M \backslash\left\{y_{1}, \ldots, y_{\beta}\right\}\right) \\
& =L\left(\bar{f}^{n}\right)+\operatorname{ind}\left(\bar{f}^{n}, \partial M \backslash\left\{y_{1}, \ldots, y_{\beta}\right\}\right) .
\end{aligned}
$$

Thus, we get that ind $\left(\bar{f}^{n}, \partial M \backslash\left\{y_{1}, \ldots, y_{\beta}\right\}\right)=0$ for $n \mid r$, so we may apply the Cancelling Procedure (Lemma 2.7) to $\bar{f}$ to get a homotopy that removes all other $r$-periodic points in $\partial M$ and such that it is constant in $\operatorname{cl}\left(B_{1} \cup \cdots \cup B_{\beta}\right)$.

Step 2. We realize $c_{i}(n)$ in the homotopy class of the initial map as indices of $g_{i}^{n}$ near the boundary fixed points $y_{i}$ in the whole $M$.

First, we extend the homotopy used in the Cancelling Procedure (in STEP 1) from $\partial M$ onto $M$. Since $f(x, t)=(\bar{f}(x), t)$ near the boundary, the above deformation of $\bar{f}$ extends to a homotopy $G_{s}$ of the pair $(M, \partial M)$.

Now, we take $f:=G_{1}$ and use Corollary 2.9 to $f$ and $g_{i}$ (for $i=1, \ldots, \beta$ ). This gives us a homotopy $H_{\tau}$ with the support $\operatorname{cl}\left(B_{1} \cup \cdots \cup B_{\beta}\right) \times[0, \varepsilon]$, preserving $\partial M$ and joining $f$ with a map which is equal to $g_{i}$ in a neighborhood of each $y_{i}$ in $M$. Let us notice (see the proof of Lemma 2.8) that $H_{\tau}$ for each $(x, t)$, is either constant or is a convex combination of $f(x, t)$ and $g_{i}(x, t)$. Since for $\tau=0$ the maps $\bar{f}$ and $\bar{g}_{i}$ are equal in each $B_{i}$, the deformation is constant in $\partial M$. Thus we have obtained in the homotopy class (preserving the boundary) of the initial map, a map $f:(M, \partial M) \rightarrow(M, \partial M)$ with exactly $\beta r$-periodic points in $\partial M$, which is $C^{1}$ in some neighborhood of each $y_{i}$. Moreover, each $y_{i}$ realizes $\operatorname{ind}\left(\bar{f}^{n}, y_{i}\right)=\operatorname{ind}\left(\bar{g}_{i}^{n}, y_{i}\right)=\bar{c}_{i}(n)$, ind $\left(f^{n}, y_{i}\right)=\operatorname{ind}\left(g_{i}^{n}, y_{i}\right)=c_{i}(n)$.

Step 3. Minimization of the number of $r$-periodic points in $\operatorname{Int} M$.

Now, we fix points $\left\{x_{1}, \ldots, x_{\alpha}\right\} \in \operatorname{Int} M$. We will show that there is a homotopy constant near $\partial M$ joining $f$ and $h$ and such that $\operatorname{Fix}\left(h^{r}\right)=\left\{x_{1}, \ldots, x_{\alpha}\right\} \cup\left\{y_{1}, \ldots, y_{\beta}\right\}$.

Since each $d_{j}$ is a $D D^{m+1}(1 \mid r)$ sequence, we may assume (applying the Creating Procedure) that $\operatorname{ind}\left(f^{n}, x_{j}\right)=d_{j}(n)$ for $n \mid r, j=1, \ldots, \alpha$. Now, analogously to (2.7) we will use the Cancelling Procedure for the whole $M$, the map $f$ and the invariant set $\left\{x_{1}, \ldots, x_{\alpha}\right\} \cup\left\{y_{1}, \ldots, y_{\beta}\right\}$.

$$
\begin{aligned}
L\left(f^{n}\right) & =\operatorname{ind}\left(f^{n},\left\{x_{1}, \ldots, x_{\alpha}\right\} \cup\left\{y_{1}, \ldots, y_{\beta}\right\}\right)+\operatorname{ind}\left(f^{n}, M \backslash\left(\left\{x_{1}, \ldots, x_{\alpha}\right\} \cup\left\{y_{1}, \ldots, y_{\beta}\right\}\right)\right) \\
& =\sum_{j=1}^{\alpha} \operatorname{ind}\left(f^{n}, x_{j}\right)+\sum_{i=1}^{\beta} \operatorname{ind}\left(f^{n}, y_{i}\right)+\operatorname{ind}\left(f^{n}, M \backslash\left(\left\{x_{1}, \ldots, x_{\alpha}\right\} \cup\left\{y_{1}, \ldots, y_{\beta}\right\}\right)\right)
\end{aligned}
$$




$$
\begin{aligned}
& =\sum_{j=1}^{\alpha} d_{j}(n)+\sum_{i=1}^{\beta} c_{i}(n)+\operatorname{ind}\left(f^{n}, M \backslash\left(\left\{x_{1}, \ldots, x_{\alpha}\right\} \cup\left\{y_{1}, \ldots, y_{\beta}\right\}\right)\right) \\
& =L\left(f^{n}\right)+\operatorname{ind}\left(f^{n}, M \backslash\left(\left\{x_{1}, \ldots, x_{\alpha}\right\} \cup\left\{y_{1}, \ldots, y_{\beta}\right\}\right)\right) .
\end{aligned}
$$

As a consequence, we get that ind $\left(f^{n}, M \backslash\left\{x_{1}, \ldots, x_{\alpha}, y_{1}, \ldots, y_{\beta}\right\}\right)=0$ for $n \mid r$. Now we apply the Cancelling Procedure. It allows us to remove all other $r$-periodic points in Int $M$. Furthermore, we can do this by a homotopy constant near the boundary. This results from the fact that the homotopy used in the Cancelling Procedure changes its values only in some small neighborhoods of some arcs joining $r$-periodic points (cf. [19] for details).

Finally, we get a $C^{1}$ map of a pair $(h, \bar{h}):(M, \partial M) \rightarrow(M, \partial M)$ homotopic to the given $(f, \bar{f})$ with $\operatorname{Fix}\left(h^{r}\right)=\left\{x_{1}, \ldots, x_{\alpha}, y_{1}, \ldots, y_{\beta}\right\}=S$, as required. The map $h$ is a $C^{1}$ map in some neighborhood $W$ of $S$ and $h^{r}$ has no fixed points outside $W$. Thus, if $h$ is not $C^{1}$ as the global map, we may approximate it by a $C^{1}$ map preserving the boundary, which is equal to $h$ on $W$ without adding any new $r$-periodic points in the compact set $M \backslash W$.

\section{Fixed point indices of iterations for a boundary fixed point}

The effective calculation of the invariant $D_{r}(f ; M, \partial M)$ is possible only if one knows all the forms of $D D^{m+1}(1 \mid r)$ sequences and $\partial D D^{m}(1 \mid r)$ pairs of sequences which appear in Definition 2.5. The complete list of $D D^{m}$ (1) sequences for arbitrary $m$ was given in [20] (Theorem 3.12 below). Our main aim in this section is to find all possible $\partial D D^{m}(1)$ pairs of sequences (Problem 3.1). The answer is given in Theorem 3.15.

Problem 3.1 Which pairs of sequences of integers are admissible as indices of iterations of a map of a pair given in $(2.2)$ at $(0,0)$ for odd iterations, i.e. what are the possible forms of $\left\{\operatorname{ind}\left(f^{n},(0,0)\right)\right\}_{n}$ and $\left\{\operatorname{ind}\left(\bar{f}^{n}, 0\right)\right\}_{n}$, where $n$ is odd.

\subsection{Periodicity of the indices for a boundary fixed point}

The classical result of Shub and Sullivan from [21] states that for an isolated fixed point $x_{0}$ of a $C^{1}$ self-map $f$ of $\mathbb{R}^{m}$ the sequence $\left\{\operatorname{ind}\left(f^{n}, x_{0}\right)\right\}_{n}$ is periodic. In this subsection we extend this result to a boundary fixed point.

Let us recall

Lemma 3.2 (Proposition 3.1 in [22]) Let $f: \mathbb{R}^{s} \rightarrow \mathbb{R}^{s}$ be a $C^{1}$ map with $f(0)=0$ and let us denote $D=D_{0} f$, the derivative of $f$ at 0 .

(1) If 0 is an isolated fixed point of $f$ then

$$
x-f^{a}(x)=\left(\sum_{j=0}^{a-1} D^{j}\right)(x-f(x))+o(\|x-f(x)\|) .
$$

(2) If 0 is an isolated fixed point for some $f^{k}$, where $k \geq 1$ and $\sum_{j=0}^{a-1} D^{j k}$ is nonsingular for an $a \geq 1$, then 0 is an isolated fixed point of $f^{a k}$ and moreover

$$
\operatorname{ind}\left(f^{a k}, 0\right)=\sigma \cdot \operatorname{ind}\left(f^{k}, 0\right),
$$

where $\sigma=\operatorname{sign} \operatorname{det}\left(\sum_{j=0}^{a-1} D^{j k}\right)$.

We will show that the formula (3.1) in the above lemma is valid for a boundary fixed point. 
Lemma 3.3 Let $f: \mathbb{R}_{+}^{m+1} \rightarrow \mathbb{R}_{+}^{m+1}$ be a $C^{1}$ map such that $f\left(\mathbb{R}_{0}^{m+1}\right) \subset \mathbb{R}_{0}^{m+1}$ and let $k \mid n$ be two numbers such that $(0,0)$ is an isolated fixed point of $f^{k}$ and $\sum_{j=0}^{n / k-1} D^{j k}$ is nonsingular. Then $(0,0)$ is an isolated fixed point of $f^{n}$ and

$$
\operatorname{ind}\left(f^{n},(0,0)\right)=\sigma \cdot \operatorname{ind}\left(f^{k},(0,0)\right),
$$

where $\sigma=\operatorname{sign} \operatorname{det}\left(\sum_{j=0}^{n / k-1} D^{j k}\right)$.

Proof It is enough to prove the lemma for $k=1$. Since $f$ maps $\left(\mathbb{R}_{+}^{m+1}, \mathbb{R}_{0}^{m+1}\right)$ into itself, $\frac{\partial f_{m+1}}{\partial t}(0,0) \geq 0$ and we will consider three cases.

- Case $\frac{\partial f_{m+1}}{\partial t}<1$.

Then for any fixed $n$ and $\tau$ the homotopy $H_{\tau}(x, t)=f^{n}(x, t \cdot \tau)$ has no fixed point different from $(0,0)$ in a neighborhood of $(0,0)$. We get:

$$
\begin{aligned}
\operatorname{ind}\left(f^{n}(x, t),(0,0)\right) & =\operatorname{ind}\left(f^{n}(x, 0),(0,0)\right)=\sigma \cdot \operatorname{ind}(f(x, 0),(0,0)) \\
& =\sigma \cdot \operatorname{ind}(f(x, t),(0,0)),
\end{aligned}
$$

where the middle equality comes from part (2) of Lemma 3.2 for $s=m$.

- Case $\frac{\partial f_{m+1}}{\partial t}>1$.

Then for any fixed $n$ the homotopy $H_{\tau}(x, t)=f^{n}(x, t+\tau)$ removes all $n$-periodic points of $f$ near $(0,0)$ and both sides of the equality (3.2) are equal to zero.

- Case $\frac{\partial f_{m+1}}{\partial t}=1$. This case needs more comment.

We recall that ind $\left(f^{n},(0,0)\right)=\operatorname{deg}\left(\mathrm{id}-\tilde{f}^{n}\right)$ (the topological degree at $\left.(0,0)\right)$, where $\tilde{f}(x, t)=f(x, \max (t, 0))$ is the composition of $f$ with the retraction $\rho: \mathbb{R}^{m+1} \rightarrow \mathbb{R}_{+}^{m+1}$.

Let us define $E(x, t)$ by the equality:

$$
(x, t)-\tilde{f}^{n}(x, t)=\left(\sum_{j=0}^{n-1} D^{j}\right)((x, t)-\tilde{f}(x, t))+E(x, t) .
$$

We claim that the homotopy

$$
H_{\tau}(x, t)=\left(\sum_{j=0}^{n-1} D^{j}\right)((x, t)-\tilde{f}(x, t))+\tau \cdot E(x, t)
$$

has no zeroes on the boundary of a sufficiently small ball $K$ centered at $(0,0) \in \mathbb{R}^{m+1}$. This implies our lemma, because by the homotopy invariance and multiplicativity property of the degree we get:

$$
\begin{aligned}
\operatorname{ind}\left(f^{n},(0,0)\right) & =\operatorname{deg}\left(\operatorname{id}-\tilde{f}^{n}\right)=\operatorname{deg}\left(H_{1}\right)=\operatorname{deg}\left(H_{0}\right) \\
& =\operatorname{deg}\left(\sum_{j=0}^{n-1} D^{j}\right) \operatorname{deg}(\operatorname{id}-\tilde{f})=\operatorname{sign}\left(\operatorname{det} \sum_{j=0}^{n-1} D^{j}\right) \cdot \operatorname{ind}(f,(0,0)) .
\end{aligned}
$$

Now we prove the claim. If $t \geq 0$ then we may follow the argument used in the proof of part (1) of Lemma 3.2 in dimension $s=m+1$, to get $E(x, t)=o(\|(x, t)-\tilde{f}(x, t)\|)$. On the other hand,

$$
\left\|\left(\sum_{j=0}^{n-1} D^{j}\right)((x, t)-\tilde{f}(x, t))\right\| \geq \varepsilon \cdot\|(x, t)-\tilde{f}(x, t)\|,
$$


where $\varepsilon>0$ is the least norm of eigenvalues of $\sum_{j=0}^{n-1} D^{j}$. Now the homotopy $H_{\tau}$ has no zeroes on the boundary of a ball $K$ (for $t \geq 0$ ) on which $\|E(x, t)\|<\varepsilon\|(x, t)-\tilde{f}(x, t)\|$.

Now let $t<0$. Then (3.3) takes the form

$$
(x, t)-f^{n}(x, 0)=\left(\sum_{j=0}^{n-1} D^{j}\right)((x, t)-f(x, 0))+E(x, t) .
$$

We will show that the homotopy $H_{\tau}$ has no zeroes different from $(0,0)$ on $K$.

Since $D=\left[\begin{array}{cc}A & \cdot \\ 0 & \frac{\partial f_{m+1}}{\partial t}\end{array}\right]$ and $\frac{\partial f_{m+1}}{\partial t}=1, \sum_{j=0}^{n-1} D^{j}=\left[\begin{array}{cc}\cdot & \cdot \\ 0 & n\end{array}\right]$.

Notice that the last coordinate of (3.4) has the form

$$
t=n \cdot t+\pi_{t}(E(x, t)),
$$

where $\pi_{t}: \mathbb{R}^{m+1} \rightarrow \mathbb{R}$ is the projection on the last coordinate. This implies $\pi_{t}(E(x, t))=$ $t(1-n)$. As a consequence, the last coordinate of the homotopy

$$
H_{\tau}(x, t)=\left(\sum_{j=0}^{n-1} D^{j}\right)((x, t)-\tilde{f}(x, t))+\tau \cdot E(x, t)
$$

equals $t(n+\tau(1-n))$. It remains to notice that this expression is negative for $t<0$, $0 \leq \tau \leq 1$. In particular, the homotopy has no zeroes for $t<0$.

Remark 3.4 Let us fix $n, k \in \mathbb{N}$ so that $l=n / k$ is odd. Then by Lemma (3.2.29) in [19] (for $n:=l-1)$ we get in Lemma $3.3 \sigma=\operatorname{sign} \operatorname{det}\left(\sum_{j=0}^{n / k-1} D^{j k}\right)=1$. Thus for an odd $n$ and its arbitrary divisor $k$ we get that the formula (3.2) takes the form:

$$
\operatorname{ind}\left(f^{n},(0,0)\right)=\operatorname{ind}(f,(0,0)) .
$$

Remark 3.5 We noticed in the proof of Lemma 3.3 that the derivative of $f$ has the form

$$
D=\left[\begin{array}{cc}
A & \cdot \\
0 & \frac{\partial f_{m+1}}{\partial t}
\end{array}\right],
$$

where $A$ is $m \times m$ matrix equal to $D_{0} \bar{f}$ the derivative of $\bar{f}$ at $0 \in \mathbb{R}_{0}^{m+1} \approx \mathbb{R}^{m}$.

\subsection{Periodic expansion of indices for a boundary fixed point}

This subsection is devoted to finding more detailed description of the forms of fixed point indices for a boundary fixed point in terms of so-called periodic expansion, i.e. by representing $\left\{\operatorname{ind}\left(f^{n},(0,0)\right)\right\}_{n}$ as a combination of some simple periodic sequences.

Definition 3.6 For a given $k \in \mathbb{N}$ we define the basic sequence $\left(\operatorname{reg}_{k}\right)$ :

$$
\operatorname{reg}_{k}(n)=\left\{\begin{array}{lll}
k & \text { if } & k \mid n, \\
0 & \text { if } & k \nmid n
\end{array}\right.
$$

Thus, $\operatorname{reg}_{k}$ is a periodic sequence:

$$
(0, \ldots, 0, k, 0, \ldots, 0, k, \ldots),
$$

where non-zero entries appear for indices which are divisible by $k$. 
By $\mu$ we will denote the classical Möbius function, i.e., $\mu: \mathbb{N} \rightarrow \mathbb{Z}$ is defined by the following three properties: $\mu(1)=1, \mu(k)=(-1)^{s}$ if $k$ is a product of $s$ different primes, $\mu(k)=0$ otherwise.

Theorem 3.7 (cf. [19]) A sequence $\left\{\operatorname{ind}\left(f^{n},(0,0)\right)\right\}_{n=1}^{\infty}$ can be represented uniquely in the following form of a periodic expansion:

$$
\operatorname{ind}\left(f^{n},(0,0)\right)=\sum_{k=1}^{\infty} a_{k} \operatorname{reg}_{k}(n)
$$

where

$$
a_{n}=\frac{1}{n} \sum_{k \mid n} \mu\left(\frac{n}{k}\right) \operatorname{ind}\left(f^{k},(0,0)\right) .
$$

Now we ask which periodic sequences may appear in the periodic expansion of $\left\{\operatorname{ind}\left(f^{n},(0,0)\right)\right\}_{n}$ for a $C^{1}$ map $f$ and a boundary fixed point $(0,0)$.

Definition 3.8 Let $H$ be a finite subset of natural numbers, by $\operatorname{LCM}(H)$ we mean the least common multiple of all elements in $H$ with the convention that $\operatorname{LCM}(\emptyset)=1$.

Definition 3.9 By $\Delta$ we will denote the set of degrees of all primitive roots of unity which are contained in $\sigma\left(D_{(0,0)} f\right)$, the spectrum of the derivative of $f$ at $(0,0)$.

Theorem 3.10 Let $f:\left(\mathbb{R}_{+}^{m+1}, \mathbb{R}_{0}^{m+1}\right) \rightarrow\left(\mathbb{R}_{+}^{m+1}, \mathbb{R}_{0}^{m+1}\right)$ be a $C^{1}$ map with $(0,0) \in$ $\mathbb{R}^{m} \times \mathbb{R}_{+}$a fixed point isolated for each iteration. Assume that $n$ is odd natural number. Then, for some integers $a_{k}$, there is the equality:

$$
\operatorname{ind}\left(f^{n},(0,0)\right)=\sum_{k \in O} a_{k} \operatorname{reg}_{k}(n),
$$

where $O=\{\operatorname{LCM}(Q): Q \subset \Delta\}$.

Proof We fix an $n \notin O$ and show that $a_{n}=0$. Let us denote $q_{k}=\operatorname{lcm}\{s \in O: s \mid k\}$. We notice that $q_{k}=\operatorname{lcm}\{s \in \Delta: s \mid k\}$.

As the sum $\sum_{j=0}^{k / q_{k}-1} D^{j q_{k}}$ is nonsingular (cf. [21]), we may apply Lemma 3.3 to $f^{q_{k}}$ and get (by Remark $3.4 \sigma=+1$ ):

$$
\operatorname{ind}\left(f^{k},(0,0)\right)=\operatorname{ind}\left(f^{q_{k}},(0,0)\right) .
$$

By the formula (3.6) and the equality (3.8) we obtain:

$$
a_{n} \cdot n=\sum_{k \mid n} \mu(n / k) \operatorname{ind}\left(f^{k},(0,0)\right)=\sum_{k \mid n} \mu(n / k) \operatorname{ind}\left(f^{q_{k}},(0,0)\right) .
$$

Thus

$$
a_{n} \cdot n=\sum_{q} \operatorname{ind}\left(f^{q},(0,0)\right)\left(\sum_{k \mid n, q_{k}=q} \mu(n / k)\right) .
$$

Finally, we use the following fact proved in [19] (Lemma (3.2.34)). Let $n, q \in \mathbb{N}$ and $n \notin O$ then

$$
\sum_{k \mid n, q_{k}=q} \mu(n / k)=0
$$

which gives the desire equality. 
Remark 3.11 For a $C^{1}$ map $\bar{f}: \mathbb{R}^{m} \rightarrow \mathbb{R}^{m}$, with 0 as an isolated fixed point for each iteration, the formula for $\left\{\operatorname{ind}\left(\bar{f}^{n}, 0\right)\right\}_{n}$ ( $n$ odd) is (except for some restriction on $a_{1}$ ) exactly the same as (3.7) with $\Delta$ replaced by $\Delta_{0}$ which is equal to the set of primitive roots of unity contained in $\sigma\left(D_{0} \bar{f}\right)$ (cf. [20,22,23]).

\subsection{The form of indices for an interior fixed point}

In this section we recall the description of possible forms of indices for a fixed point in an open subset of $\mathbb{R}^{m}$ given in [20,22]. We only consider the case of odd iterations, and thus the forms of indices are simpler. We use the following notation: for natural $s$ we denote by $L(s)$ any set of natural numbers of the form $\{\operatorname{LCM}(Q): Q \subset L, \# L=s$ and $1 \notin L\}$.

Theorem 3.12 Let $f$ be a $C^{1}$ self-map of $\mathbb{R}^{m}$, where $m \geq 3$, having 0 as an isolated fixed point for each iteration. Then,

- any sequence of local indices of iterations $\left\{\operatorname{ind}\left(f^{n}, 0\right)\right\}_{n=1}^{\infty}$ for odd $n$ has one of the forms given below in (I) for $m$ odd and (II) for $m$ even.

- Every sequence of integers which is of one of the forms listed below can be realized as a sequence of local indices of iterations of a $C^{1}$ self-map of $\mathbb{R}^{m}$.

(I) For $m$ odd

$$
\begin{aligned}
\left(A^{o}\right) \operatorname{ind}\left(f^{n}, 0\right) & =\sum_{k \in L\left(\frac{m-3}{2}\right)} a_{k} \operatorname{reg}_{k}(n) . \\
\left(B^{o}\right),\left(C^{o}\right),\left(D^{o}\right) \operatorname{ind}\left(f^{n}, 0\right) & =\sum_{k \in L\left(\frac{m-1}{2}\right)} a_{k} \operatorname{reg}_{k}(n),
\end{aligned}
$$

where

$$
a_{1}=\left\{\begin{array}{cl}
1 & \text { in the case }\left(B^{o}\right) \\
-1 & \text { in the case }\left(C^{o}\right) \\
0 & \text { in the case }\left(D^{o}\right)
\end{array}\right.
$$

(II) For $m$ even:

$$
\begin{aligned}
& \left(B^{e}\right) \text { ind }\left(f^{n}, 0\right)=\sum_{k \in L\left(\frac{m-2}{2}\right)} a_{k} \operatorname{reg}_{k}(n) . \\
& \left(F^{e}\right) \text { ind }\left(f^{n}, 0\right)=\sum_{k \in L\left(\frac{m}{2}\right)} a_{k} \operatorname{reg}_{k}(n),
\end{aligned}
$$

where $a_{1}=1$.

3.4 The form of indices for a map of a pair (f, $\overline{\mathbf{f}}):\left(\mathbb{R}_{+}^{\mathrm{m}+1}, \mathbb{R}_{0}^{\mathrm{m}+1}\right)$

$\rightarrow\left(\mathbb{R}_{+}^{\mathbf{m}+1}, \mathbb{R}_{0}^{\mathbf{m}+1}\right)$

In this section we will solve Problem 3.1. First, we formulate a statement which shows a relation between the index of a boundary map $\bar{f}$ and its extension $f$, which we will use in the proof of Theorem 3.15. 
Lemma 3.13 (Theorem 5.1 in [9]) Let $(f, \bar{f}):\left(\mathbb{R}_{+}^{m+1}, \mathbb{R}_{0}^{m+1}\right) \rightarrow\left(\mathbb{R}_{+}^{m+1}, \mathbb{R}_{0}^{m+1}\right)$ be a $C^{1}$ map of a pair with $(0,0) \in \mathbb{R}^{m} \times \mathbb{R}_{+}=\mathbb{R}_{+}^{m+1}$ an isolated fixed point of $f$. Assume that +1 is not an eigenvalue of $D_{0} \bar{f}$ i.e. $I-D_{0} \bar{f}$ is an isomorphism.

Then

$$
\operatorname{ind}(f,(0,0))=\operatorname{ind}(\bar{f}, 0) \text { or } \operatorname{ind}(f,(0,0))=0 .
$$

We will also make use of the following lemma.

Lemma 3.14 (cf. [24]) Let $Y=A \cup B$ be a topological space, $x_{0} \in A \cap B, U$ be an open neighborhood of $x_{0}$ in $Y$. Let $F: U \rightarrow Y, F(U \cap A) \subset A$ and $F(U \cap B) \subset B$.

If $x_{0}$ is an isolated fixed point of $F$ and $U, U \cap A, U \cap B$ and $U \cap A \cap B$ are ENRs, then:

$$
\operatorname{ind}\left(F, x_{0}\right)+\operatorname{ind}_{A \cap B}\left(F, x_{0}\right)=\operatorname{ind}_{A}\left(F, x_{0}\right)+\operatorname{ind}_{B}\left(F, x_{0}\right),
$$

where by $\operatorname{ind}_{G}\left(F, x_{0}\right)$ we denote $\operatorname{ind}\left(F_{\mid G}, x_{0}\right)$.

Theorem 3.15 Let $(f, \bar{f}):\left(\mathbb{R}_{+}^{m+1}, \mathbb{R}_{0}^{m+1}\right) \rightarrow\left(\mathbb{R}_{+}^{m+1}, \mathbb{R}_{0}^{m+1}\right)$ be a $C^{1}$ map of a pair with $(0,0) \in \mathbb{R}^{m} \times \mathbb{R}_{+}$an isolated fixed point for each iteration and $m \geq 3$. Then,

(1) any pair of sequences of local indices of iterations $\left\{\operatorname{ind}\left(f^{n},(0,0)\right)\right\}_{n}$ and $\left\{\operatorname{ind}\left(\bar{f}^{n}, 0\right)\right\}_{n}$ for odd $n$ has one of the forms given below in (I) for $m$ odd and (II) for $m$ even (with $\left.a_{k}, b_{k} \in \mathbb{Z}\right)$.

(2) Every pair of sequences of integers which is of one of the forms listed below can be realized as a pair of sequences of local indices of iterations of a $C^{1}$ map of a pair $(f, \bar{f}):\left(\mathbb{R}_{+}^{m+1}, \mathbb{R}_{0}^{m+1}\right) \rightarrow\left(\mathbb{R}_{+}^{m+1}, \mathbb{R}_{0}^{m+1}\right)$.

(I) For $m$ odd

$$
\begin{aligned}
\left(A^{o}\right) \operatorname{ind}\left(\bar{f}^{n}, 0\right) & =\sum_{k \in L\left(\frac{m-3}{2}\right)} a_{k} \operatorname{reg}_{k}(n), \\
\operatorname{ind}\left(f^{n},(0,0)\right) & =\sum_{k \in L\left(\frac{m-3}{2}\right)} b_{k} \operatorname{reg}_{k}(n) . \\
\left(B^{o}\right),\left(C^{o}\right),\left(D^{o}\right) \operatorname{ind}\left(\bar{f}^{n}, 0\right) & =\sum_{k \in L\left(\frac{m-1}{2}\right)} a_{k} \operatorname{reg}_{k}(n), \\
\operatorname{ind}\left(f^{n},(0,0)\right) & =\sum_{k \in L\left(\frac{m-1}{2}\right)} b_{k} \operatorname{reg}_{k}(n),
\end{aligned}
$$

where $b_{1}$ could be arbitrary and

$$
a_{1}=\left\{\begin{array}{cl}
1 & \text { in the case }\left(B^{o}\right) \\
-1 & \text { in the case }\left(C^{o}\right) \\
0 & \text { in the case }\left(D^{o}\right)
\end{array}\right.
$$

(II) For $m$ even:

$$
\begin{aligned}
\left(B^{e}\right) \operatorname{ind}\left(\bar{f}^{n}, 0\right) & =\sum_{k \in L\left(\frac{m-2}{2}\right)} a_{k} \operatorname{reg}_{k}(n), \quad \operatorname{ind}\left(f^{n},(0,0)\right)=\sum_{k \in L\left(\frac{m-2}{2}\right)} b_{k} \operatorname{reg}_{k}(n), \\
\left(F_{\partial_{0}}^{e}\right),\left(F_{\partial_{1}}^{e}\right) \quad \operatorname{ind}\left(\bar{f}^{n}, 0\right) & =\sum_{k \in L\left(\frac{m}{2}\right)} a_{k} \operatorname{reg}_{k}(n), \quad \operatorname{ind}\left(f^{n},(0,0)\right)=\sum_{k \in L\left(\frac{m}{2}\right)} b_{k} \operatorname{reg}_{k}(n),
\end{aligned}
$$


where $a_{1}=1$, and

$$
b_{1}= \begin{cases}0 & \text { in the case }\left(F_{\partial_{0}}^{e}\right), \\ 1 & \text { in the case }\left(F_{\partial_{1}}^{e}\right) .\end{cases}
$$

Remark 3.16 Notice that according to Theorem $3.15 a_{k}$ and $b_{k}$, the coefficients in periodic expansions of ind $\left(f^{n},(0,0)\right)_{n}$ and $\left\{\operatorname{ind}\left(\bar{f}^{n}, 0\right)\right\}_{n}$, except for $a_{1}$ and $b_{1}$, could be any (also different) integers.

Proof of Theorem 3.15 (1) Theorem 3.12 describes the forms of indices for $\bar{f}$, $\left\{\operatorname{ind}\left(\bar{f}^{n}, 0\right)\right\}_{n}$. On the other hand, by Theorem 3.10 and Remark 3.11 the form of $\left\{\operatorname{ind}\left(f^{n},(0,0)\right)\right\}_{n}$ (where $\bar{f}$ is the restriction of $f$ to the boundary) is the same, as $D_{(0,0)} f$ has only one real nonnegative eigenvalue more than $D_{0} \bar{f}$ (by Remark 3.5) and thus $\Delta=\Delta_{0}$ or $\Delta=\Delta_{0} \cup\{1\}$, so $\{\operatorname{LCM}(Q): Q \subset \Delta\}=\left\{\operatorname{LCM}(Q): Q \subset \Delta_{0}\right\}$. (We will show in part (2) of the proof that there are no further restrictions i.e. that each pair of indices of that form can be realized by a $C^{1}$ map of a pair). The only condition we must verify are the restrictions for $b_{1}$ in the cases $\left(F_{\partial_{0}}^{e}\right)$ and $\left(F_{\partial_{1}}^{e}\right)$.

Assume, without lost of generality, that there are $2 s$ (where $2 s=m$ ) different pairs of eigenvalues of $D_{0} \bar{f}$, which are primitive roots of unity of degree $\geq 3$ (otherwise the sequences of indices have simpler form and we are in the other case). Thus $\left\{\operatorname{ind}\left(\bar{f}^{n}, 0\right)\right\}_{n}$ by Theorem 3.12 is of the form $\left(F^{e}\right)$ and $a_{1}=1$. On the other hand, by the dimension argument $1 \notin \sigma\left(D_{0} \bar{f}\right)$, and then by Lemma $3.13 b_{1}=a_{1}$ or $b_{1}=0$, which ends the proof of the restrictions for $a_{1}$ and $b_{1}$ and the proof of part (1).

Proof of Theorem 3.15 (2) Now we show that each pair of sequences listed in (I) and (II) can be realized by a $C^{1}$ map of a pair. We indicate realizations in dependence on $s$, the number of primitive roots of unity of degree $\geq 3$ in $\Delta=\Delta_{0}$.

(A) $m=2 s$.

We will realize the pairs of maps given in $\left(F_{\partial_{0}}^{e}\right)$ and $\left(F_{\partial_{1}}^{e}\right)$.

Definition 3.17 We define some subspaces of $\mathbb{R}_{+}^{m+1}$ :

- $W=\left\{(x, t) \in \mathbb{R}_{+}^{m+1}:\|x\|=t\right\}$,

- $V_{0}=\left\{(x, t) \in \mathbb{R}_{+}^{m+1}: x=0\right\}$,

- $V=\left\{(x, t) \in \mathbb{R}_{+}^{m+1}: t=0\right\}$.

We will use methods similar to those used in Section 9 in [20]. We will define a map on each of the spaces listed in Definition 3.17 as a discretization of some smooth flow. It is possible to extend this maps to a $C^{1}$ self-map of the whole $\mathbb{R}_{+}^{m+1}$ with 0 as the only periodic point, in such a way that the fixed point indices of all iterations for the extension are the same as for the restriction to $W \cup V_{0} \cup V$ (cf. [20] for details).

By the formula (3.12) applied to $\left(W \cup V_{0}\right) \cup V$, taking into account that the index on the singleton $\{(0,0)\}$ is equal to 1 , we get

$$
\operatorname{ind}\left(f^{n},(0,0)\right)=\operatorname{ind}_{W}\left(f^{n},(0,0)\right)+\operatorname{ind}_{V_{0}}\left(f^{n},(0,0)\right)+\operatorname{ind}_{V}\left(f^{n},(0,0)\right)-2 \operatorname{reg}_{1}(n) .
$$


We define the self-maps on the spaces $W, V_{0}, V$ in the following way.

Consider $V=\mathbb{R}_{0}^{m+1} \approx \mathbb{R}^{m}$. By Remark 3.11 and by Theorem 3.12 we can find a self-map of $\mathbb{R}_{0}^{m+1}$ (of the type $F^{e}$ ) such that its indices are equal to $\sum_{k \in L\left(\frac{m}{2}\right)} a_{k} \operatorname{reg}_{k}(n)$, where $a_{1}=1$.

On $W \approx \mathbb{R}_{0}^{m+1} \approx \mathbb{R}^{m}$ we repeat the same construction with the coefficients $c_{k}=b_{k}-a_{k}$, so that $\operatorname{ind}_{W}\left(f^{n},(0,0)\right)=\sum_{k \in L\left(\frac{m}{2}\right)} c_{k} \operatorname{reg}_{k}(n)$, where $c_{1}=1$.

On $V_{0}$, which is a half-line, we define (in an obvious way) $f$ so that either $\operatorname{ind}_{V_{0}}\left(f^{n},(0,0)\right)=0$ or $\operatorname{ind}_{V_{0}}\left(f^{n},(0,0)\right)=\operatorname{reg}_{1}(n)$. Thus we obtain two maps, which indices may be expressed in dependence on a parameter $\alpha$ as ind $V_{0}\left(f^{n},(0,0)\right)=\alpha \operatorname{reg}_{1}(n)$, where $\alpha \in\{0,1\}$.

Finally, substituting obtained values of indices to (3.13), we get

$$
\operatorname{ind}\left(f^{n},(0,0)\right)=\alpha \operatorname{reg}_{1}(n)+\sum_{k \in L\left(\frac{m}{2}\right)} b_{k} \operatorname{reg}_{k}(n),
$$

where $\alpha \in\{0,1\}$. In this way we constructed two kinds of maps, one of the type $\left(F_{\partial_{0}}^{e}\right)$ for $\alpha=0$ and the second of the type $\left(F_{\partial_{1}}^{e}\right)$ for $\alpha=1$, which completes the construction in the case (A).

(B) $m=2 s+1$.

We will realize the pairs of maps given in $B^{o}, C^{o}$ and $D^{o}$.

We represent $\mathbb{R}_{0}^{m+1}=\mathbb{R}^{m}$ as the product of subspaces $V \times V^{\prime}$ where $\operatorname{dim} V=2 s$, $\operatorname{dim} V^{\prime}=1$. Then $\mathbb{R}_{+}^{m+1}=\left(V \times V^{\prime}\right) \times \mathbb{R}_{+}$. Now, on the subspace $V \times \mathbb{R}_{+}$, in the same way as in the case (A), we can find a map of a pair $(f, \bar{f})$ whose indices have basic sequences $\operatorname{reg}_{k}$ with arbitrary coefficients $a_{k}, b_{k}$ for $k \geq 3, k \in L\left(\frac{m-1}{2}\right)$. We have still some room,

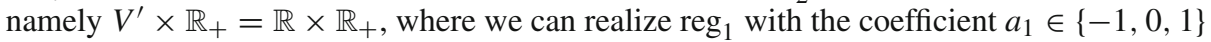
and arbitrary $b_{1}$, which will complete the proof of part (B). The last realization can be done, due to the following lemma.

Lemma 3.18 For any pair of integer numbers $a_{1}, b_{1}$ with $\left|a_{1}\right| \leq 1$ there exists a $C^{1}$ map of a pair $(f, \bar{f}):\left(\mathbb{R}_{+}^{2}, \mathbb{R}_{0}^{2}\right) \rightarrow\left(\mathbb{R}_{+}^{2}, \mathbb{R}_{0}^{2}\right)$, where $(0,0) \in \mathbb{R} \times \mathbb{R}_{+}$is an isolated fixed point for each iteration of $f$ and

$$
\operatorname{ind}\left(\bar{f}^{n}, 0\right)=a_{1}, \quad \operatorname{ind}\left(f^{n},(0,0)\right)=b_{1},
$$

for each $n \in \mathbb{N}$.

Proof Let us define three types of smooth flows defined on $\mathbb{R}_{0}^{2}=\mathbb{R}$ with 0 as the only stationary point, and consider indices of its discretization $\bar{f}$.

(1) The flow such that 0 is a sink, then ind $\left(\bar{f}^{n}, 0\right)=1$.

(2) The flow such that 0 is a source, then ind $\left(\bar{f}^{n}, 0\right)=-1$.

(3) The flow such that 0 is a stationary point removable by any small perturbation, then $\operatorname{ind}\left(\bar{f}^{n}, 0\right)=0$.

Now, let us consider planar flows $h_{p}: \mathbb{R} \times \mathbb{R}_{+} \times \mathbb{R} \rightarrow \mathbb{R} \times \mathbb{R}_{+}$with phase portraits consisting of $2|p|$ hyperbolic regions for $p<0,2 p$ elliptic regions for $p>0$ (an example of such flow is given in Fig. 1). The flow may be taken as smooth as we like, in particular with the discretization being $C^{1}$ map (see [25]).

We take its time-one map and denote it by $h_{p}(1)$. Next, we consider $f_{p}$, the self-map of $\mathbb{R}^{2}$ equal to $h_{p}(1)$ composed with retraction $\rho: \mathbb{R}^{2} \rightarrow \mathbb{R}_{+}^{2}$.

We calculate ind $\left(f_{p},(0,0)\right)$ as the number of revolutions of the vector connecting $z$ with $f(z)$ for $z \in C$ running along a small circle $C$ centered at $(0,0)$. Notice that the fact that 
Fig. 1 An example of the flow $h_{p}$ with $p=-2$

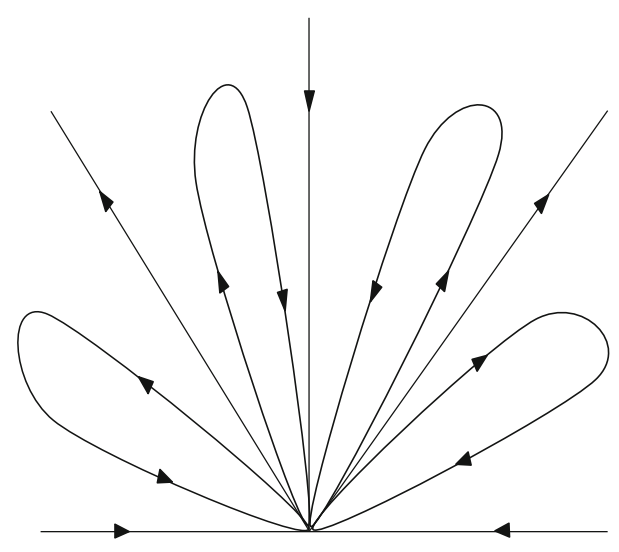

Fig. 2 The situation from the proof of Lemma 3.18 part (3)

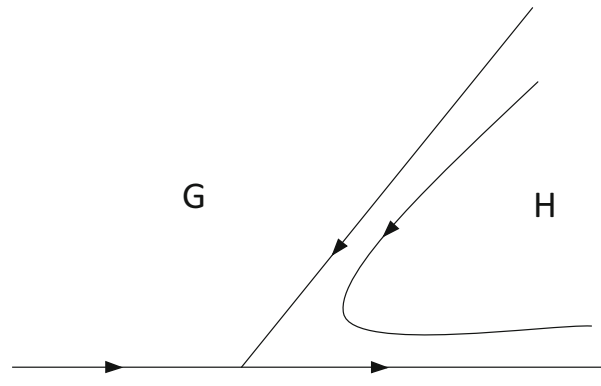

$h_{p}^{n}(1)=h_{p}(n)$ implies ind $\left(f_{p}^{n}(0,0)\right)=\operatorname{ind}\left(f_{p},(0,0)\right)$. Now in each of the three cases (1)-(3) we will define an extension of $\bar{f}$ with arbitrary integer index.

(1) We take the extension equal to $h_{p}$ for $p \neq 0$ and a 2 -dimensional sink on $\mathbb{R} \times \mathbb{R}_{+}$in case $p=0$. Then we get

$$
\operatorname{ind}\left(f_{p},(0,0)\right)=\left\{\begin{array}{cl}
1-|p| & \text { if } p<0 \\
1+p & \text { if } p>0 \\
1 & \text { if } p=0
\end{array}\right.
$$

(2) We take the extension equal to $h_{p}$ for $p \neq 0$ and a 2-dimensional source on $\mathbb{R} \times \mathbb{R}_{+}$in case $p=0$. Then we get

$$
\operatorname{ind}\left(f_{p},(0,0)\right)=\left\{\begin{array}{cl}
-|p| & \text { if } p<0, \\
p & \text { if } p>0, \\
0 & \text { if } p=0 .
\end{array}\right.
$$

(3) Consider the situation presented in Fig. 2. We take the extension equal to $h_{p}$ (for $p \neq 0$ ) in the area denoted by $G$ and one hyperbolic sector in the area denoted by $H$. For $p=0$ we take 2-dimensional sink on $\mathbb{R} \times \mathbb{R}_{+}$. Then we get

$$
\operatorname{ind}\left(f_{p},(0,0)\right)=\left\{\begin{array}{cl}
-|p| & \text { if } p<0, \\
p & \text { if } p>0, \\
0 & \text { if } p=0
\end{array}\right.
$$

(C) $m \geq 2 s+2$. We will realize the pairs of maps given in $\left(A^{o}\right)$ and $\left(B^{e}\right)$. 
We have a similar decomposition $\mathbb{R}_{+}^{m+1}=\left(V \times V^{\prime}\right) \times \mathbb{R}_{+}$, where $\operatorname{dim} V=2 s, \operatorname{dim} V^{\prime} \geq 2$. Again, we may find a map of a pair $(f, \bar{f})$ such that on the subspace $V \times \mathbb{R}_{+}$we can realize indices having basic sequences $\operatorname{reg}_{k}$ with arbitrary coefficients $a_{k}, b_{k}$ for $k \geq 3$ and $k \in L\left(\frac{m-2}{2}\right)$ for $m$ even or $k \in L\left(\frac{m-3}{2}\right)$ for $k$ odd. Furthermore, on $V^{\prime} \times \mathbb{R}_{+}=\mathbb{R}^{2} \times \mathbb{R}^{+}$we can realize sequences $a_{1} \operatorname{reg}_{1}$ for $\bar{f}$ and $b_{1} \operatorname{reg}_{1}$ for $f$ with arbitrary $a_{1}, b_{1}$ (cf. [25]).

\section{Reducing $r$-periodic points to one point}

In this section we illustrate the whole theory, finding necessary and sufficient conditions to reduce $r$-periodic points in the $C^{1}$-homotopy class just to one point (Theorem 4.1 ) and applying the result to self-maps of 6-dimensional closed ball (Theorem 4.2).

Theorem 4.1 Let $M$ be a smooth compact and simply-connected manifold with simplyconnected boundary $\partial M, r$ be an odd natural number and $(f, \bar{f}):(M, \partial M) \rightarrow(M, \partial M)$. Assume $\left\{L\left(f^{n}\right)\right\}_{n \mid r}$ is not constantly equal to 0 . Then there exists a map of a pair $(g, \bar{g})$ : $(M, \partial M) \rightarrow(M, \partial M) C^{1}$-homotopic to $(f, \bar{f})$ such that $\operatorname{Fix}\left(g^{r}\right)=\{p\}$ if and only if one of the following conditions is satisfied:

(1) $L\left(\bar{f}^{n}\right)=0$ for all $r \mid n$ and $\left(L\left(f^{n}\right)\right)_{n}$ is a $D D^{m+1}(1 \mid r)$ sequence,

(2) the pair $\left(L\left(f^{n}\right), L\left(\bar{f}^{n}\right)\right)_{n}$ is a $\partial D D^{m}(1 \mid r)$ pair of sequences.

Proof By Definition 2.5 of $D_{r}(f ; M, \partial M)$ we decompose Lefschetz numbers of iterations of $f$ and $\bar{f}$ into the minimal sum $\alpha+\beta$ of $\alpha D D^{m+1}(1 \mid r)$ sequences and $\beta \partial D D^{m}(1 \mid r)$ pairs of sequences.

Thus $D_{r}(f ; M, \partial M)=1$ iff

(A) $\alpha=1$ and $\beta=0$, or

(B) $\alpha=0$ and $\beta=1$.

In the case (A) $\beta=0$ is equivalent to $L\left(\bar{f}^{n}\right)=0$ for all $r \mid n$. On the other hand, $\alpha=1$ is equivalent to the fact that $\left(L\left(f^{n}\right)\right)_{n}$ is itself a $D D^{m+1}(1 \mid r)$ sequence, which gives the condition (1).

The condition (B) may be expressed in the following form: for $n \mid r$

$$
L\left(\bar{f}^{n}\right)=\bar{c}_{1}(n) \text { and } L\left(f^{n}\right)=c_{1}(n),
$$

where $\left(c_{1}, \bar{c}_{1}\right)$ is a $\partial D D^{m}(1 \mid r)$ pair of sequences, so (B) is equivalent to (2).

Theorem 4.2 Let $B^{6} \subset \mathbb{R}^{6}$ be a closed 6-dimensional ball with the boundary $\partial B^{6}=S^{5}$. Consider a $C^{1}$ map of a pair $(f, \bar{f}):\left(B^{6}, S^{5}\right) \rightarrow\left(B^{6}, S^{5}\right)$. Let $D$ denote the degree of a self-map $\bar{f}$ of $S^{5}$ and $r$ be a fixed odd natural number. Then, it is possible to reduce the number of r-periodic points in the $C^{1}$-homotopy class of $(f, \bar{f})$ to one point if and only if one of the following conditions is satisfied:

(a) $D \in\{-1,0,1\}$,

(b) $r$ is a prime number or $r=1$,

(c) $D=2$ and $\left(r=p q\right.$ or $\left.r=p^{2}\right)$, where $p$, $q$ are prime numbers.

Proof As $m=5$ is odd here, we get that $L\left(\bar{f}^{n}\right)=1-D^{n}$. Furthermore $L\left(f^{n}\right)=1$, since $B^{6}$ is contractible. First, notice that (1) of Theorem 4.1 is equivalent to $D=1$, because $L\left(f^{n}\right)=\operatorname{reg}_{1}(n)$ is a $D D^{m+1}(1 \mid r)$ sequence for any $m$ and $r$. 
Next, we show that for the rest of cases (a), (b), (c) listed in the thesis of Theorem 4.2 (i.e. for $D \neq 1$ ) the condition (2) of Theorem 4.1 is satisfied i.e. that $\left(\operatorname{reg}_{1}(n), 1-D^{n}\right)_{n \mid r}$ is a $\partial D D^{5}(1 \mid r)$ pair of sequences.

We get by Theorem 3.15 (part (I)) the following list of $\partial D D^{5}(1)$ pairs of sequences for odd divisors (by $[d, l]$ we denote the least common multiple of $d$ and $l$ ):

$(\alpha) \bar{c}=a_{1} \operatorname{reg}_{1}(n)+a_{d} \operatorname{reg}_{k}(n), c=b_{1} \operatorname{reg}_{1}(n)+b_{d} \operatorname{reg}_{d}(n) ;$ for $a_{1}, a_{d}, b_{1}, b_{d} \in \mathbb{Z}$.

$(\beta) \bar{c}=\delta \operatorname{reg}_{1}(n)+a_{d} \operatorname{reg}_{d}(n)+a_{l} \operatorname{reg}_{l}(n)+a_{[d, l]} \operatorname{reg}_{[d, l]}(n), c=b_{1} \operatorname{reg}_{1}(n)+b_{d} \operatorname{reg}_{d}(n)+$ $b_{l} \operatorname{reg}_{l}(n)+b_{[d, l]} \operatorname{reg}_{[d, l]}(n)$; for $\delta \in\{-1,0,1\}, a_{d}, a_{l}, a_{[d, l]}, b_{1}, b_{d}, b_{l}, b_{[d, l]} \in \mathbb{Z}, d, l \geq$ 3.

Case (a). Let us notice that for $D=0$ or $D=-1$ we get, for each odd $r$, constant sequences:

$$
\left(L\left(f^{n}\right), L\left(\bar{f}^{n}\right)\right)_{n \mid r}= \begin{cases}\left(\operatorname{reg}_{1}(n), \operatorname{reg}_{1}(n)\right)_{n \mid r} & \text { if } D=0 \\ \left(\operatorname{reg}_{1}(n), 2 \operatorname{reg}_{1}(n)\right)_{n \mid r} & \text { if } D=-1\end{cases}
$$

each of which is obviously a $\partial D D^{5}(1 \mid r)$ pair of sequences of the type $(\alpha)$.

Case (b). If $r$ is a prime number, then $\left(L\left(f^{n}\right), L\left(\bar{f}^{n}\right)\right)_{n \mid r}=\left(\operatorname{reg}_{1}(n), a_{1} \operatorname{reg}_{1}(n)+\right.$ $\left.a_{p} \operatorname{reg}_{p}(n)\right)_{n \mid r}$ (similarly for $r=1$, with $a_{p}=0$ ), so it is again a $\partial D D^{5}(1 \mid r)$ pair of sequences of the type $(\alpha)$.

Case (c). Assume $D=2$, then $a_{1}=L(f)=1-D=-1$. Thus

$$
\begin{aligned}
& \left(L\left(f^{n}\right), L\left(\bar{f}^{n}\right)\right)_{n \mid r} \\
& \quad= \begin{cases}\left(\operatorname{reg}_{1}(n),-\operatorname{reg}_{1}(n)+a_{p} \operatorname{reg}_{p}(n)+a_{q} \operatorname{reg}_{q}(n)+a_{p q} \operatorname{reg}_{p q}(n)\right)_{n \mid r} & \text { if } r=p q, \\
\left(\operatorname{reg}_{1}(n),-\operatorname{reg}_{1}(n)+a_{p} \operatorname{reg}_{p}(n)+a_{p^{2}} \operatorname{reg}_{p^{2}}(n)\right)_{n \mid r} & \text { if } r=p^{2} .\end{cases}
\end{aligned}
$$

If $r=p q$ then $[p, q]=p q$, and as a consequence in both cases $\left(L\left(f^{n}\right), L\left(\bar{f}^{n}\right)\right)_{n \mid r}$ is a $\partial D D^{5}(1 \mid r)$ pair of sequences of the type $(\beta)$ with $\delta=-1$.

To complete the proof we have to show that if none of the conditions (a), (b), (c) of Theorem 4.2 is satisfied then $\left(L\left(f^{n}\right), L\left(\bar{f}^{n}\right)\right)_{n \mid r}$ is not a $\partial D D^{5}(1 \mid r)$ pair of sequences.

Take $|D| \geq 2$ and assume that $r$ has at least two nontrivial divisors, $s$ and $t$. It is known ([26], Theorem 1.2) that for $|D| \geq 2$ each $a_{n}$, the $n$th coefficient of the periodic expansion of $\left\{L\left(\bar{f}^{n}\right)\right\}_{n=1}^{\infty}$, is non-zero. Taking into account that $a_{1}=1-D$ we observe that in the periodic expansion of $\left\{L\left(\bar{f}^{n}\right)\right\}_{n \mid r}$ must appear the following non-zero terms:

$$
(1-D) \operatorname{reg}_{1}(n)+a_{s} \operatorname{reg}_{s}(n)+a_{t} \operatorname{reg}_{t}(n)+a_{[s, t]} \operatorname{reg}_{[s, t]}(n) \text {. }
$$

Thus $\left(L\left(f^{n}\right), L\left(\bar{f}^{n}\right)\right)_{n \mid r}$ could be a $\partial D D^{5}(1 \mid r)$ pair of sequences only if it is of the type $(\beta)$ with $\delta=1-D \in\{-1,0,1\}$, thus for $D=2$ and only if there are no more then two different non-trivial divisors.

Acknowledgements Funding was provided by National Science Centre, Poland (Grant No. UMO-2014/15/B/ ST1/01710).

Open Access This article is distributed under the terms of the Creative Commons Attribution 4.0 International License (http://creativecommons.org/licenses/by/4.0/), which permits unrestricted use, distribution, and reproduction in any medium, provided you give appropriate credit to the original author(s) and the source, provide a link to the Creative Commons license, and indicate if changes were made. 


\section{References}

1. Dugardein, G-J.: Nielsen periodic point theory on infra-nilmanifolds. arXiv:1508.04345

2. Fel'shtyn, A., Lee, J.B.: The Nielsen and Reidemeister numbers of maps on infra-solvmanifolds of type (R). Topol. Appl. 181, 62-103 (2015)

3. Hart, E., Heath, P., Keppelmann, E.: Algorithms for Nielsen type periodic numbers of maps with remnant on surfaces with boundary and on bouquets of circles. I. Fundam. Math. 200(2), 101-132 (2008)

4. Jezierski, J.: Homotopy periodic sets of selfmaps of real projective spaces. Bol. Soc. Mat. Mex. (3) 11(2), 294-302 (2005)

5. Kim, H.J., Lee, J.B., Yoo, W.S.: Computation of the Nielsen type numbers for maps on the Klein bottle. J. Korean Math. Soc. 45(5), 1483-1503 (2008)

6. Lee, J.B., Zhao, X.: Nielsen type numbers and homotopy minimal periods for maps on 3-solvmanifolds. Algebra. Geom. Topol. 8(1), 563-580 (2008)

7. Lee, J.B., Zhao, X.: Nielsen type numbers and homotopy minimal periods for maps on the 3-nilmanifolds. Sci. China Ser. A 51(3), 351-360 (2008)

8. Graff, G., Jezierski, J.: Minimizing the number of periodic points for smooth maps. Non-simply connected case. Topol. Appl. 158(3), 276-290 (2011)

9. Brown, R., Greene, R., Schirmer, H.: Fixed points of map extensions. In: Topological Fixed Point Theory and Applications (Tianjin, 1988), Lecture Notes in Math., vol. 1411, pp. 24-45. Springer, Berlin (1989)

10. Brown, R.F., Greene, R.E.: An interior fixed point property of the disc. Am. Math. Mon. 101(1), 39-47 (1994)

11. Khamsemanan, N., Brown, R.F., Lee, C., Dhompongsa, S.: A fixed point theorem for smooth extension maps. Fixed Point Theory Appl. 2014, 97 (2014). doi:10.1186/1687-1812-2014-97

12. Khamsemanan, N., Brown, R.F., Lee, C., Dhompongsa, S.: Interior fixed points of unit-sphere-preserving euclidean maps. Fixed Point Theory Appl. 2012, 183 (2012). doi:10.1186/1687-1812-2012-183

13. Schirmer, H.: Nielsen theory of transversal fixed point sets. Fundam. Math. 141(1), 31-59 (1992)

14. Jiang, B.J.: Fixed point classes from a differential viewpoint. In: Lecture Notes in Math., vol. 886, pp. 163-170. Springer (1981)

15. Graff, G., Jezierski, J.: Minimal number of periodic points for $C^{1}$ self-maps of compact simply-connected manifolds. Forum Math. 21(3), 491-509 (2009)

16. Dold, A.: Fixed point indices of iterated maps. Invent. Math. 74, 419-435 (1983)

17. Graff, G., Jezierski, J.: Combinatorial scheme of finding minimal number of periodic points for smooth self-maps of simply connected manifolds. J. Fixed Point Theory Appl. 13(1), 63-84 (2012)

18. Jezierski, J.: Wecken's theorem for periodic points in dimension at least 3. Topol. Appl. 153(11), 18251837 (2006)

19. Jezierski, J., Marzantowicz, W.: Homotopy methods in topological fixed and periodic points theory. In: Topological Fixed Point Theory and Its Applications, vol. 3. Springer, Dordrecht (2006)

20. Graff, G., Jezierski, J., Nowak-Przygodzki, P.: Fixed point indices of iterated smooth maps in arbitrary dimension. J. Differ. Equ. 251, 1526-1548 (2011)

21. Shub, M., Sullivan, P.: A remark on the Lefschetz fixed point formula for differentiable maps. Topology 13, 189-191 (1974)

22. Chow, S.N., Mallet-Paret, J., Yorke, J. A.: A periodic orbit index which is a bifurcation invariant. In: Geometric Dynamics (Rio de Janeiro, 1981), Lecture Notes in Math., vol. 1007, pp. 109-131. Springer, Berlin (1983)

23. Babenko, I.K., Bogatyi, S.A.: The behavior of the index of periodic points under iterations of a mapping. Math. USSR Izv. 38, 1-26 (1992)

24. O’Neill, B.: Essential sets and fixed points. Am. J. Math. 75, 497-509 (1953)

25. Graff, G., Nowak-Przygodzki, P.: Fixed point indices of iterations of $C^{1}$ maps in $\mathbb{R}^{3}$. Discrete Cont. Dyn. Syst. 16(4), 843-856 (2006)

26. Llibre, J., Paranõs, J., Rodriguez, J.A.: Periods for transversal maps on compact manifolds with a given homology. Houst. J. Math. 24(3), 397-407 (1998) 